\title{
On stress integration of coupled viscodamage- viscoplasticity models with separate yield/loading surfaces
}

\author{
Timo Saksala
}

Summary. This paper deals with numerical integration of stresses, inelastic strains and internal variables related to coupled viscodamage-viscoplasticity models. The class of models considered here is the one where the viscodamage and viscoplasticity parts are described independently based on their specific loading/yield criteria and evolutions laws. Moreover, in the viscodamage part, an anisotropic compliance damage formulation is adopted. Both the viscodamage and the viscoplasticity components are formulated in terms of the consistency model by Wang (1997). Two methods for coupling the damage and the plasticity parts are presented and their performance compared. In the first more traditional method, both models are solved simultaneously returning the trial stress onto the intersection of the criteria while updating the internal variables. The second, nonstandard method exploits the damage strain to impose iteratively the stress equality on the stress vectors returned independently on the respective, viscodamage and viscoplasticity surfaces. A special emphasis is laid on the treatment of the corner point plasticity case. After the general treatment, the two methods are illustrated with an application to the Mohr-Coulomb viscoplasticity model combined with Rankine viscodamage model.

Key words: coupled viscodamage-viscoplasticity model, stress integration, damage strain

Received 21 May 2018. Accepted 11 October 2018. Published online 24 December 2018.

\section{Introduction}

Numerical analyses of engineering structures and materials involve constitutive modeling of failing materials. The two main classes of the constitutive models employed therein are the plasticity and damage models [1-3]. On the one hand, pure plasticity models can capture the observed inelastic strains and strength degradation (of brittle materials) but not the stiffness degradation. On the other hand, plain damage models capture the stiffness and strength degradation but not the inelastic (plastic) strains. Therefore, the most realistic description of materials, such as concrete and rocks, is achieved by coupled damage-plasticity models, as exemplified by Refs. [4-7]. These approaches are illustrated schematically in Figure 1. Moreover, many engineering materials exhibit strong strain rate sensitivity under dynamic loading. Thereby, the

Corresponding author: timo.saksala@tut.fi 
coupled viscodamage-viscoplasticity models seem the most suitable approach for applications involving dynamic loadings.
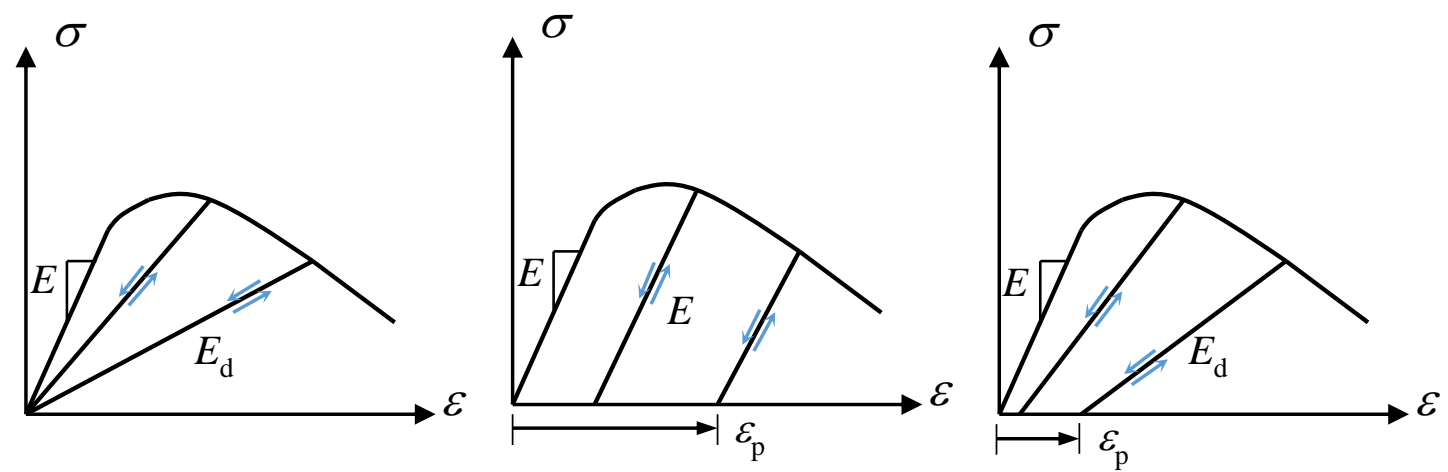

Figure 1. Schematic illustration of different approaches: pure damage model (left), pure plasticity model (middle) and combined damage-plasticity model (right).

There are several ways to couple the damage and plasticity parts of the combined model [3-9] and to perform the stress integration and updates of the internal variables. If the damage model is formulated with a loading function that indicates stress or strain states leading to damage evolution, the classical corner plasticity techniques from the multisurface plasticity theory can be employed [5, 7-9]. However, if damage is driven by plastic strains then no loading function is needed since the yield function indicates also the stress states leading to damaging [4]. Finally, a less standard method exists that exploits the damage strain to impose iteratively the equality of the stresses returned independently on the respective damage and plasticity surfaces [6].

In the present paper, the classical corner plasticity method based on consistency conditions and the damage strain based iteration method are employed and applied to the class of combined viscodamage-viscoplasticity models. A special emphasis is laid on the treatment of a corner plasticity case, i.e. the return onto the intersection of the criteria. Moreover, the compliance damage concept is adopted. The viscosity is accommodated by the Wang's consistent viscoplasticity format which enables the employment of classical return mapping algorithms [10]. After the general treatment of the problem, the developed methods are illustrated with a numerical example taken from constitutive modelling of rock. More specifically, in the model the Mohr-Coulomb viscoplasticity describes the material failure in compression while a Rankine type of viscodamage model governs the rate-dependent damage processes in tension.

It is emphasized here that the two integration methods are previously presented in the literature so that the main novelty of the present work is their comparison. However, studies concerning the nonstandard method based on iterative imposition of the stresses exploiting the damage strain are extremely rare - the author could find only two works $[3,6]$. Moreover, in both of these previous studies the considered constitutive models were rate independent while in the present paper the models are rate dependent. Therefore, as this method proves, in the numerical examples presented here, to be efficient in solving problems involving rate dependent damage and plasticity, the present work has a justified goal of promoting this method. 


\section{Coupled viscodamage-viscoplasticity model}

The fundamental assumption of the models considered here is the additive decomposition, valid under the infinitesimal strain theory, of the total strain, $\boldsymbol{\varepsilon}$, in to elastic, viscoplastic and viscodamage parts, $\boldsymbol{\varepsilon}_{\mathrm{e}}, \boldsymbol{\varepsilon}_{\mathrm{vp}}, \boldsymbol{\varepsilon}_{\mathrm{vd}}$, respectively, by

$$
\boldsymbol{\varepsilon}=\boldsymbol{\varepsilon}_{\mathrm{e}}+\boldsymbol{\varepsilon}_{\mathrm{vp}}+\boldsymbol{\varepsilon}_{\mathrm{vd}} .
$$

Different strain components of the decomposition (1) are illustrated in Figure 2 in a typical cyclic loading of an elasto-viscoplastic-viscodamaging material. It should be noted that the viscodamage strain, which is a result of micromechanisms such as void and crack formation, is recoverable upon unloading.

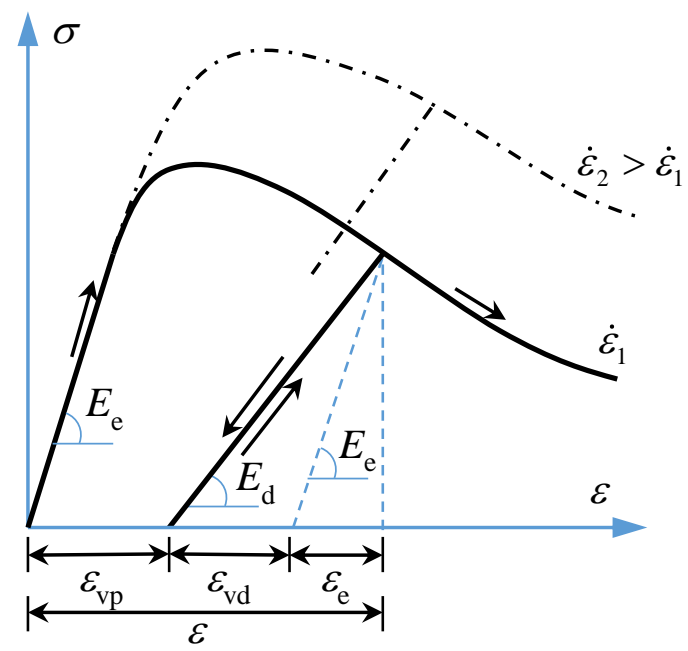

Figure 2. Schematic illustration for the decomposition of strain into different components under cyclic loading of an elasto-viscodamage-viscoplastic material.

\section{Viscoplastic consistency model}

Unlike the classical overstress viscoplasticity formulations by Perzyna and DuvautLions, the more recent viscoplastic consistency model [10] utilizes the consistency condition recasting the viscoplasticity into a format that allows to use the standard computational plasticity algorithms for stress integration. The main components of such a model are

$$
\begin{aligned}
& f_{\mathrm{vp}}=f\left(\boldsymbol{\sigma}, \kappa_{\mathrm{vp}}, \dot{\kappa}_{\mathrm{vp}}\right)=\hat{f}_{\mathrm{vp}}(\boldsymbol{\sigma})-\left(\sigma_{y}+h_{\mathrm{vp}} \kappa_{\mathrm{vp}}+s_{\mathrm{vp}} \dot{\kappa}_{\mathrm{vp}}\right) \\
& \dot{\boldsymbol{\varepsilon}}_{\mathrm{vp}}=\dot{\lambda}_{\mathrm{vp}} \frac{\partial g_{\mathrm{vp}}}{\partial \boldsymbol{\sigma}}, \quad \dot{\kappa}_{\mathrm{vp}}=\dot{\lambda}_{\mathrm{vp}} k_{\mathrm{vp}}\left(\boldsymbol{\sigma}, \kappa_{\mathrm{vp}}\right) \\
& f_{\mathrm{vp}} \leq 0, \quad \dot{\lambda}_{\mathrm{vp}} \geq 0, \quad \dot{\lambda}_{\mathrm{vp}} f_{\mathrm{vp}}=0
\end{aligned}
$$

where $f_{\mathrm{vp}}$ is the dynamic yield function, $\hat{f}_{\mathrm{vp}}(\boldsymbol{\sigma})$ is the given function of stress $\sigma, \sigma_{y}$ is the static yield stress, $h_{\mathrm{vp}}$ and $s_{\mathrm{vp}}$ are the plastic and viscosity moduli, $\mathrm{g}_{\mathrm{vp}}$ is the viscoplastic 
potential, $\kappa_{\mathrm{vp}}, \dot{\kappa}_{\mathrm{vp}}$ are the internal variable and its rate, respectively, $\dot{\lambda}_{\mathrm{vp}}$ is the viscoplastic increment, and $k_{\mathrm{vp}}\left(\boldsymbol{\sigma}, \kappa_{\mathrm{vp}}\right)$ is function that relates $\kappa_{\mathrm{vp}}$ to $\lambda_{\mathrm{vp}}$.

Thermodynamics poses some restrictions on the plastic potential as well as on function $k_{\mathrm{vp}}$. Accordingly, if $g_{\mathrm{vp}}$ is a convex function possessing the property $g_{\mathrm{vp}}\left(\boldsymbol{\sigma}, K_{\mathrm{vp}}\right)-g_{\mathrm{vp}}(\mathbf{0}, 0) \geq 0$ for $\forall \boldsymbol{\sigma}, K_{\mathrm{vp}}$ such that $f_{\mathrm{vp}}\left(\boldsymbol{\sigma}, K_{\mathrm{vp}}, \dot{\kappa}_{\mathrm{vp}}\right)=0$, then the dissipation inequality

$$
\boldsymbol{\sigma}: \dot{\boldsymbol{\varepsilon}}_{\mathrm{vp}}-K_{\mathrm{vp}} \dot{\kappa}_{\mathrm{vp}} \geq 0
$$

(with $K_{\mathrm{vp}}=h_{\mathrm{vp}} \kappa_{\mathrm{vp}}$ ) is fulfilled for the following evolution laws

$$
\dot{\boldsymbol{\varepsilon}}_{\mathrm{vp}}=\dot{\lambda}_{\mathrm{vp}} \frac{\partial g_{\mathrm{vp}}}{\partial \boldsymbol{\sigma}}, \quad \dot{\boldsymbol{\kappa}}_{\mathrm{vp}}=-\dot{\lambda}_{\mathrm{vp}} \frac{\partial g_{\mathrm{vp}}}{\partial K_{\mathrm{vp}}}
$$

By comparing (2) and (3), function $k_{\mathrm{vp}}$ is identified as $k_{\mathrm{vp}}\left(\boldsymbol{\sigma}, \kappa_{\mathrm{vp}}\right)=-\partial g_{\mathrm{vp}} / \partial K_{\mathrm{vp}}$.

\section{Viscodamage consistency model: compliance tensor approach}

The compliance damage approach (see [3]) based on the usage of the elastic compliance tensor as an anisotropic damage variable is chosen here. Due to the formal equivalence between the compliance damage theory and plasticity theory, this model can be recast into the consistency viscosity format - hence the name viscodamage consistency model. The main ingredients of such a model are [7]

$$
\begin{aligned}
& f_{\mathrm{vd}}=f\left(\boldsymbol{\sigma}, \kappa_{\mathrm{vd}}, \dot{\kappa}_{\mathrm{vd}}\right)=\hat{f}_{\mathrm{vd}}(\boldsymbol{\sigma})-\left(\sigma_{f}+h_{\mathrm{vd}} \kappa_{\mathrm{vd}}+s_{\mathrm{vd}} \dot{\kappa}_{\mathrm{vd}}\right) \\
& \dot{\mathbf{D}}=\frac{\dot{\lambda}_{\mathrm{vd}}}{\hat{f}_{\mathrm{vd}}(\boldsymbol{\sigma})} \frac{\partial f_{\mathrm{vd}}}{\partial \boldsymbol{\sigma}} \otimes \frac{\partial f_{\mathrm{vd}}}{\partial \boldsymbol{\sigma}}, \quad \dot{\kappa}_{\mathrm{vd}}=\dot{\lambda}_{\mathrm{vd}} k_{\mathrm{vd}}\left(\boldsymbol{\sigma}, \kappa_{\mathrm{vd}}\right) \\
& f_{\mathrm{vd}} \leq 0, \quad \dot{\lambda}_{\mathrm{vd}} \geq 0, \quad \dot{\lambda}_{\mathrm{vd}} f_{\mathrm{vd}}=0
\end{aligned}
$$

where $f_{\mathrm{vd}}$ is the viscodamage loading function, $\sigma_{f}$ is the fracture stress indicating the damage initiation stress, $\mathbf{D}$ is the (fourth order) compliance tensor, whereas the meanings of the rest of symbols are equivalent to the corresponding ones related to the viscoplastic model.

It should be noted that the evolution law for the compliance tensor in (5) is derived by assuming that the damage loading function $\hat{f}_{\mathrm{vd}}(\boldsymbol{\sigma})$ is a homogeneous function of degree one, i.e. so that $\partial \hat{f}_{\mathrm{vd}}(\boldsymbol{\sigma}) / \partial \boldsymbol{\sigma}: \boldsymbol{\sigma}=\hat{f}_{\mathrm{vd}}(\boldsymbol{\sigma})$. The model accounts for the loadinginduced anisotropy through the tensor product $(\otimes)$ of the damage loading function gradients.

The mechanical dissipation inequality for this model reads 


$$
\frac{1}{2} \boldsymbol{\sigma}: \dot{\mathbf{D}}: \boldsymbol{\sigma}-K_{\mathrm{vd}} \dot{K}_{\mathrm{vd}} \geq 0
$$

with $K_{\mathrm{vd}}=h_{\mathrm{vd}} K_{\mathrm{vd}}$. For softening behavior, the second term of the dissipation inequality is non-positive while the non-negativity of the first term follows trivially from tensor algebra. Thus, the model verifies the dissipation inequality.

In the compliance damage approach, the current value of compliance tensor, which evolves by damage from the initial value $\mathbf{D}_{(t=0)}=\mathbf{0}$, is added to the elastic compliance, $\mathbf{E}_{\mathrm{d}}=\left(\mathbf{C}_{\mathrm{e}}^{-1}+\mathbf{D}\right)^{-1}$, where $\mathbf{E}_{\mathrm{d}}$ is the damaged stiffness and $\mathbf{C}_{\mathrm{e}}$ is the standard elasticity tensor. The relation connecting the damaged compliance and the damage strain is $\boldsymbol{\varepsilon}_{\mathrm{vd}}=\mathbf{D}: \boldsymbol{\sigma}$. Finally, as the focus of the present paper is on the stress integration methods, the unilateral conditions of damage, i.e. the stiffness recovery upon closing microcracks, are neglected in the present work. They are dealt with, e.g. in [7].

\section{Stress return mapping algorithms for coupled models}

Two different methods for stress integration are described here. Both are essentially based on the standard elastic predictor-plastic corrector split widely used in computational plasticity $[2,3]$. However, due to the presence of the damage strain in the decomposition (1), the elastic predictor stress is slightly different from the standard one:

$$
\boldsymbol{\sigma}_{n+1}^{\text {trial }}=\mathbf{C}_{\mathrm{e}}:\left(\boldsymbol{\varepsilon}_{n+1}^{(i)}-\boldsymbol{\varepsilon}_{n}^{\mathrm{vp}}-\boldsymbol{\varepsilon}_{n+1}^{\mathrm{vd},(k=0)}\right)=\underbrace{\left(\mathbf{C}_{\mathrm{e}}^{-1}+\mathbf{D}_{n}\right)^{-1}}_{\mathbf{E}_{\mathrm{d}}}:\left(\boldsymbol{\varepsilon}_{n+1}^{(i)}-\boldsymbol{\varepsilon}_{n}^{\mathrm{vp}}\right),
$$

where the relation just mentioned above, $\boldsymbol{\varepsilon}_{n+1}^{\mathrm{vd}(k=0)}=\mathbf{D}_{n}: \boldsymbol{\sigma}_{n+1}^{\text {trial }}$, has been used, while $i$ and $n$ denote the global and local iterations, respectively. In the following, the stress integration algorithms are presented for the case when both the yield and damage loading function are positive. The cases where only one criterion is active are readily identifiable from the more general case with both criteria active.

\section{Algorithm 1: direct solution of coupled damage-plasticity processes}

In the first algorithm, the viscodamage and viscoplasticity multipliers are solved directly by enforcing the consistency conditions in (2) and (5) simultaneously. After the elimination of the rate of the internal variables in the yield and damage loading functions and an expansion by the vector valued Taylor series, the following stress return mapping scheme is obtained (see [7] for details): 


$$
\begin{aligned}
& {\left[\delta \lambda_{\mathrm{vd}} \delta \lambda_{\mathrm{vp}}\right]^{\mathrm{T}}=\mathbf{G}_{n}^{-1}\left[f_{\mathrm{vd}} f_{\mathrm{vp}}\right]_{n}^{\mathrm{T}}} \\
& \boldsymbol{\varepsilon}_{n+1}^{\mathrm{vp}}=\boldsymbol{\varepsilon}_{n}^{\mathrm{vp}}+\delta \lambda_{\mathrm{vp}} \frac{\partial g_{\mathrm{vp}}}{\partial \boldsymbol{\sigma}_{n}} \\
& \mathbf{D}_{n+1}=\mathbf{D}_{n}+\frac{\delta \lambda_{\mathrm{vd}}}{\hat{f}_{\mathrm{vd}}\left(\boldsymbol{\sigma}_{n}\right)} \frac{\partial f_{\mathrm{vd}}}{\partial \boldsymbol{\sigma}_{n}} \otimes \frac{\partial f_{\mathrm{vd}}}{\partial \boldsymbol{\sigma}_{n}} \\
& \boldsymbol{\sigma}_{n+1}=\boldsymbol{\sigma}_{n}-\left(\mathbf{C}_{\mathrm{e}}^{-1}+\mathbf{D}_{n}\right)^{-1}:\left(\delta \lambda_{\mathrm{vd}} \frac{\partial f_{\mathrm{vd}}}{\partial \boldsymbol{\sigma}_{n}}+\delta \lambda_{\mathrm{vp}} \frac{\partial g_{\mathrm{vp}}}{\partial \boldsymbol{\sigma}_{n}}\right) \\
& \Delta \lambda_{\mathrm{vd}, n+1}=\Delta \lambda_{\mathrm{vd}, n}+\delta \lambda_{\mathrm{vd}}, \quad \Delta \lambda_{\mathrm{vp}, n+1}=\Delta \lambda_{\mathrm{vp}, n}+\delta \lambda_{\mathrm{vp}} \\
& \kappa_{\mathrm{vd}, n+1}=\kappa_{\mathrm{vd}, n}+k_{\mathrm{vd}}\left(h_{\mathrm{vd}, n} \delta \lambda_{\mathrm{vd}}+s_{\mathrm{vd}} \dot{\lambda}_{\mathrm{vd}, n+1}\right), \quad \dot{\lambda}_{\mathrm{vd}, n+1}=\frac{\Delta \lambda_{\mathrm{vd}, n+1}}{\Delta t} \\
& \kappa_{\mathrm{vp}, n+1}=\kappa_{\mathrm{vp}, n}+k_{\mathrm{vp}}\left(h_{\mathrm{vp}, n} \delta \lambda_{\mathrm{vp}}+s_{\mathrm{vp}} \dot{\lambda}_{\mathrm{vp}, n+1}\right), \quad \dot{\lambda}_{\mathrm{vp}, n+1}=\frac{\Delta \lambda_{\mathrm{vp}, n+1}}{\Delta t}
\end{aligned}
$$

where $[\bullet]_{n}^{\mathrm{T}}$ means that the arguments are evaluated at values of $n$th iteration, and

$$
\mathbf{G}_{n}=\left[\begin{array}{ll}
G_{11} & G_{12} \\
G_{21} & G_{22}
\end{array}\right]=\left[\begin{array}{cc}
\frac{\partial f_{\mathrm{vd}}}{\partial \boldsymbol{\sigma}_{n}}: \mathbf{E}_{\mathrm{d}}: \frac{\partial f_{\mathrm{vd}}}{\partial \boldsymbol{\sigma}_{n}}+k_{\mathrm{vd}}\left(h_{\mathrm{vd}, n}+\frac{s_{\mathrm{vd}}}{\Delta t}\right) & \frac{\partial f_{\mathrm{vd}}}{\partial \boldsymbol{\sigma}_{n}}: \mathbf{E}_{\mathrm{d}}: \frac{\partial g_{\mathrm{vp}}}{\partial \boldsymbol{\sigma}_{n}} \\
\frac{\partial f_{\mathrm{vp}}}{\partial \boldsymbol{\sigma}_{n}}: \mathbf{E}_{\mathrm{d}}: \frac{\partial f_{\mathrm{vd}}}{\partial \mathbf{\sigma}_{n}} & \frac{\partial f_{\mathrm{vp}}}{\partial \boldsymbol{\sigma}_{n}}: \mathbf{E}_{\mathrm{d}}: \frac{\partial g_{\mathrm{vp}}}{\partial \boldsymbol{\sigma}_{n}}+k_{\mathrm{vp}}\left(h_{\mathrm{vp}, n}+\frac{s_{\mathrm{vp}}}{\Delta t}\right)
\end{array}\right]
$$

in matrix form. Equations (8) are repeatedly evaluated until an acceptable tolerance is reached, i.e. $f_{\mathrm{vd}}\left(\boldsymbol{\sigma}_{n}, \kappa_{\mathrm{vd}, n}, \dot{\kappa}_{\mathrm{vd}, n}\right) \leq \mathrm{Tol}$ and $f_{\mathrm{vp}}\left(\boldsymbol{\sigma}_{n}, \kappa_{\mathrm{vp}, n}, \dot{\kappa}_{\mathrm{vp}, n}\right) \leq \mathrm{Tol}$.

Finally, the material tangent tensor is derived for this approach. The starting point is the rate form of the stress-strain relation (7) developed further as follows:

$$
\begin{aligned}
\dot{\boldsymbol{\sigma}} & =\mathbf{C}_{\mathrm{e}}:\left(\dot{\boldsymbol{\varepsilon}}-\dot{\boldsymbol{\varepsilon}}_{\mathrm{vp}}-\dot{\boldsymbol{\varepsilon}}_{\mathrm{vd}}\right) \\
& =\mathbf{C}_{\mathrm{e}}:\left(\dot{\boldsymbol{\varepsilon}}-\dot{\lambda}_{\mathrm{vp}} \frac{\partial f_{\mathrm{vp}}}{\partial \boldsymbol{\sigma}}-\dot{\mathbf{D}}: \boldsymbol{\sigma}-\mathbf{D}: \dot{\boldsymbol{\sigma}}\right) \\
& =\mathbf{C}_{\mathrm{e}}:\left(\dot{\boldsymbol{\varepsilon}}-\dot{\lambda}_{\mathrm{vp}} \frac{\partial g_{\mathrm{vp}}}{\partial \boldsymbol{\sigma}}-\frac{\dot{\lambda}_{\mathrm{vd}}}{\hat{f}_{\mathrm{vd}}(\boldsymbol{\sigma})} \frac{\partial f_{\mathrm{vd}}}{\partial \boldsymbol{\sigma}} \otimes \frac{\partial f_{\mathrm{vd}}}{\partial \boldsymbol{\sigma}}: \boldsymbol{\sigma}-\mathbf{D}: \dot{\boldsymbol{\sigma}}\right) \\
& =\left(\mathbf{C}_{\mathrm{e}}^{-1}+\mathbf{D}\right)^{-1}:\left(\dot{\boldsymbol{\varepsilon}}-\dot{\lambda}_{\mathrm{vp}} \frac{\partial g_{\mathrm{vp}}}{\partial \boldsymbol{\sigma}}-\dot{\lambda}_{\mathrm{vd}} \frac{\partial f_{\mathrm{vd}}}{\partial \boldsymbol{\sigma}}\right)
\end{aligned}
$$

where, in addition to the evolution equations for the viscoplastic strain and the compliance tensor, the tensor relation $(\mathbf{A} \otimes \mathbf{B}): \mathbf{C}=(\mathbf{B}: \mathbf{C}) \mathbf{A}$ as well as the assumption that $\hat{f}_{\mathrm{vd}}$ is a homogenous function of degree one have been used. In passing, it is noted that the stress update formula in equations (8) was derived similarly as (10).

Now, the viscoplastic multipliers are given by the first of equations (8) and, thus, the material tangent relation becomes 


$$
\begin{aligned}
& \dot{\boldsymbol{\sigma}}=\mathbf{C}_{\text {epd }}: \dot{\boldsymbol{\varepsilon}} \quad \text { with } \\
& \mathbf{C}_{\mathrm{epd}}=\mathbf{E}_{\mathrm{d}}-G_{11}^{-1} \mathbf{E}_{\mathrm{d}}: \frac{\partial f_{\mathrm{vp}}}{\partial \boldsymbol{\sigma}} \otimes \frac{\partial g_{\mathrm{vp}}}{\partial \boldsymbol{\sigma}}: \mathbf{E}_{\mathrm{d}}-G_{12}^{-1} \mathbf{E}_{\mathrm{d}}: \frac{\partial f_{\mathrm{vd}}}{\partial \boldsymbol{\sigma}} \otimes \frac{\partial g_{\mathrm{vp}}}{\partial \boldsymbol{\sigma}}: \mathbf{E}_{\mathrm{d}}+ \\
& -G_{21}^{-1} \mathbf{E}_{\mathrm{d}}: \frac{\partial f_{\mathrm{vp}}}{\partial \boldsymbol{\sigma}} \otimes \frac{\partial f_{\mathrm{vd}}}{\partial \boldsymbol{\sigma}}: \mathbf{E}_{\mathrm{d}}-G_{22}^{-1} \mathbf{E}_{\mathrm{d}}: \frac{\partial f_{\mathrm{vd}}}{\partial \boldsymbol{\sigma}} \otimes \frac{\partial f_{\mathrm{vd}}}{\partial \boldsymbol{\sigma}}: \mathbf{E}_{\mathrm{d}}
\end{aligned}
$$

where $G_{i j}^{-1}$ are the entries of the inverse of matrix in (9) and $\mathbf{E}_{\mathrm{d}}$ is as in (7).

\section{Algorithm 2: iterative imposition of stress equality using damage strain}

The basic idea of this approach is to carry out the viscodamage and viscoplasticity computations independently without any exchange of the results. This results in two stresses, viscodamage and viscoplastic stress, returned onto their respective yield and damage surfaces. Then, the equality of these stresses is enforced iteratively using the damage strain as the argument. Thereby, at the end of the local iteration, the stress should be

$$
\boldsymbol{\sigma}_{n+1}=\left\{\begin{array}{l}
\mathbf{C}_{\mathrm{e}}:\left(\boldsymbol{\varepsilon}_{n+1}^{(i)}-\boldsymbol{\varepsilon}_{n+1}^{\mathrm{vp}}-\boldsymbol{\varepsilon}_{n+1}^{\mathrm{vd},(k)}\right)=: \boldsymbol{\sigma}_{n+1}^{\mathrm{vp}} \\
\mathbf{D}_{n+1}^{-1}: \boldsymbol{\varepsilon}_{n+1}^{\mathrm{vd},(k)}=: \boldsymbol{\sigma}_{n+1}^{\mathrm{vd}}
\end{array}\right.
$$

where $k$ denotes the iteration with respect to the damage strain, $n$ is the iteration counter with respect to the separate damage and plasticity stress return mappings, and $i$ is global iteration. The trial stress is computed using equation (7) and then, the first value of the damage strain is based on that as $\boldsymbol{\varepsilon}_{n+1}^{\mathrm{vd},(0)}=\mathbf{D}_{n}^{-1}: \boldsymbol{\sigma}_{n+1}^{\text {trial }}$. However, a problem arises at the transition from elastic response to damage process if the initial value of the compliance damage tensor is set to zero, as specified above. Therefore, in this algorithm the initial compliance damage is set to $\mathbf{D}_{0}=\mathbf{C}_{\mathrm{e}}^{-1}[6]$. Nevertheless, this choice requires the elastic modulus to be doubled to retain the experimental elastic behaviour of the material.

The damage strain is updated based on the residual difference, i.e. the nonequilibrium, between the stresses as follows $[3,6]$

$$
\begin{aligned}
& \boldsymbol{\varepsilon}_{n+1}^{\mathrm{vd},(k+1)}=\boldsymbol{\varepsilon}_{n+1}^{\mathrm{vd},(k)}+\Delta \boldsymbol{\varepsilon}_{n+1}^{\mathrm{vd},(k)} \\
& \Delta \boldsymbol{\varepsilon}_{n+1}^{\mathrm{vd},(k)}=\left(\mathbf{C}_{n+1}^{\mathrm{vp}}+\mathbf{C}_{n+1}^{\mathrm{vd}}\right)^{-1}: \mathbf{r}\left(\boldsymbol{\varepsilon}_{n+1}^{\mathrm{vd},(k)}\right) \quad \text { with } \\
& \mathbf{r}\left(\boldsymbol{\varepsilon}_{n+1}^{\mathrm{vd},(k)}\right)=\boldsymbol{\sigma}_{n+1}^{\mathrm{vp}}\left(\boldsymbol{\varepsilon}_{n+1}^{\mathrm{vd},(k)}\right)-\boldsymbol{\sigma}_{n+1}^{\mathrm{vd}}\left(\boldsymbol{\varepsilon}_{n+1}^{\mathrm{vd},(k)}\right)
\end{aligned}
$$

where $\mathbf{C}_{n+1}^{\mathrm{vp}}, \mathbf{C}_{n+1}^{\mathrm{vd}}$ are the material tangents for the independently integrated viscoplasticity and viscodamage models. These are defined as [3] 


$$
\begin{gathered}
\mathbf{C}_{n+1}^{\mathrm{vp}}=\mathbf{C}_{\mathrm{e}}-\frac{\mathbf{C}_{\mathrm{e}}: \frac{\partial g_{\mathrm{vp}}}{\partial \boldsymbol{\sigma}_{n+1}} \otimes \frac{\partial f_{\mathrm{vp}}}{\partial \boldsymbol{\sigma}_{n+1}}: \mathbf{C}_{\mathrm{e}}}{\frac{\partial f_{\mathrm{vp}}}{\partial \boldsymbol{\sigma}_{n+1}}: \mathbf{C}_{\mathrm{e}}: \frac{\partial g_{\mathrm{vp}}}{\partial \boldsymbol{\sigma}_{n+1}}+k_{\mathrm{vp}}\left(h_{\mathrm{vp}, n+1}+\frac{s_{\mathrm{vp}}}{\Delta t}\right)} \\
\mathbf{C}_{n+1}^{\mathrm{vd}}=\mathbf{D}_{n+1}^{-1}-\frac{\mathbf{D}_{n+1}^{-1}: \frac{\partial f_{\mathrm{vd}}}{\partial \boldsymbol{\sigma}_{n+1}} \otimes \frac{\partial f_{\mathrm{vd}}}{\partial \boldsymbol{\sigma}_{n+1}}: \mathbf{D}_{n+1}^{-1}}{\frac{\partial f_{\mathrm{d}}}{\partial \boldsymbol{\sigma}_{n+1}}: \mathbf{D}_{n+1}^{-1}: \frac{\partial f_{\mathrm{d}}}{\partial \boldsymbol{\sigma}_{n+1}}+k_{\mathrm{vd}}\left(h_{\mathrm{vd}, n+1}+\frac{s_{\mathrm{dd}}}{\Delta t}\right)}
\end{gathered}
$$

Now, the material tangent for the coupled case can be identified because at the end of iterations (13) the stresses are equal. Thus, exploiting the results (14), one obtains

$$
\begin{aligned}
& \dot{\boldsymbol{\sigma}}_{n+1}=\dot{\boldsymbol{\sigma}}_{n+1}^{\mathrm{vp}}:=\mathbf{C}_{n+1}^{\mathrm{vp}}:\left(\dot{\boldsymbol{\varepsilon}}_{n+1}-\dot{\boldsymbol{\varepsilon}}_{n+1}^{\mathrm{vd}}\right)=\mathbf{C}_{n+1}^{\mathrm{vd}}: \dot{\boldsymbol{\varepsilon}}_{n+1}^{\mathrm{vd}}=: \dot{\boldsymbol{\sigma}}_{n+1}^{\mathrm{vd}}=\dot{\boldsymbol{\sigma}}_{n+1} \\
& \Rightarrow \dot{\boldsymbol{\sigma}}_{n+1}=\mathbf{C}_{n+1}^{\mathrm{vd}}\left(\mathbf{C}_{n+1}^{\mathrm{vp}}+\mathbf{C}_{n+1}^{\mathrm{vd}}\right)^{-1} \mathbf{C}_{n+1}^{\mathrm{vp}}: \dot{\boldsymbol{\varepsilon}}_{n+1}=\mathbf{C}_{n+1}^{\mathrm{vdp}}: \dot{\boldsymbol{\varepsilon}}_{n+1}
\end{aligned}
$$

Finally, the algorithm is sketched in Table 1.

Table 1.Algorithm for coupled viscodamage-viscoplasticity model based on the damage strain.

$$
\text { Elastic trial step: } \begin{aligned}
& \boldsymbol{\sigma}_{n+1}^{\text {trial }}=\mathbf{C}_{\mathrm{e}}:\left(\boldsymbol{\varepsilon}_{n+1}^{(i)}-\boldsymbol{\varepsilon}_{n}^{\mathrm{vp}}-\boldsymbol{\varepsilon}_{n+1}^{\mathrm{vd}(k=0)}\right)=\left(\mathbf{C}_{\mathrm{e}}^{-1}+\mathbf{D}_{n}\right)^{-1}:\left(\boldsymbol{\varepsilon}_{n+1}^{(i)}-\boldsymbol{\varepsilon}_{n}^{\mathrm{vp}}\right) \\
& \boldsymbol{\varepsilon}_{n+1}^{\mathrm{vd},(k=0)}=\mathbf{D}_{n}: \boldsymbol{\sigma}_{n+1}^{\text {trial }}
\end{aligned}
$$$$
\text { Viscoplasticity: given } \boldsymbol{\varepsilon}_{n+1}^{\mathrm{vd},(k)} \text { Do Viscodamage: given } \boldsymbol{\varepsilon}_{n+1}^{\mathrm{vd}(k)} \text { Do }
$$$$
\operatorname{IF} f_{\mathrm{vp}}^{\text {trial }}=f_{\mathrm{vp}}\left(\boldsymbol{\sigma}_{n+1}^{\text {trial }}, \kappa_{\mathrm{vp}, n}, \dot{\kappa}_{\mathrm{vp}, n}\right)<0 \quad \operatorname{IF} f_{\mathrm{vd}}^{\text {trial }}=f_{\mathrm{vd}}\left(\boldsymbol{\sigma}_{n+1}^{\text {trial }}, \kappa_{\mathrm{vd}, n}, \dot{\kappa}_{\mathrm{vd}, n}\right)<0
$$$$
\text { THEN Elastic Step } \rightarrow \text { Exit } \quad \text { THEN Elastic Step } \rightarrow \text { Exit }
$$$$
\text { ELSE Perform Viscoplastic Step } \quad \text { ELSE Perform Viscodamage Step }
$$$$
\Rightarrow \boldsymbol{\sigma}_{n+1}^{\mathrm{vp}}, \boldsymbol{\varepsilon}_{n+1}^{\mathrm{vp}}, \kappa_{\mathrm{vp}, n+1}, \lambda_{\mathrm{vp}, n+1} \quad \Rightarrow \boldsymbol{\sigma}_{n+1}^{\mathrm{vd}}, \mathbf{D}_{n+1}, \kappa_{\mathrm{vd}, n+1}, \lambda_{\mathrm{vd}, n+1}
$$

$$
\begin{aligned}
& \text { IF } \underbrace{\left\|\boldsymbol{\sigma}_{n+1}^{\mathrm{vp}}-\boldsymbol{\sigma}_{n+1}^{\mathrm{vd}}\right\|}_{\left\|\mathbf{r}\left(\varepsilon_{n+1}^{\mathrm{vd}, k}\right)\right\|}>\text { TOL Perform correction: } \\
& \begin{array}{l}
\Delta \boldsymbol{\varepsilon}_{n+1}^{\mathrm{vd},(k)}=\left(\mathbf{C}_{n+1}^{\mathrm{vp}}+\mathbf{C}_{n+1}^{\mathrm{vd}}\right)^{-1}: \mathbf{r}\left(\boldsymbol{\varepsilon}_{n+1}^{\mathrm{vd},(k)}\right) \\
\boldsymbol{\varepsilon}_{n+1}^{\mathrm{vd},(k+1)}=\boldsymbol{\varepsilon}_{n+1}^{\mathrm{vd},(k)}+\Delta \boldsymbol{\varepsilon}_{n+1}^{\mathrm{vd},(k)}
\end{array}
\end{aligned}
$$

The computations with respect to viscoplasticity and viscodamage steps in Table 1 can be performed with the steps identifiable from those of the coupled model integration given in Equation (8).

Finally, a comment on the computational labour required by these algorithms is given. It seems that the Algorithm 2 in Table 1 requires more, at least during a single iterative sweep, computational effort than Algorithm 1 due the usage of the material tangent tensors when solving the new damage strain (Equation (13)). However, 
Algorithm 1 needs the matrix $\mathbf{G}$ in (9) at each iterative sweep to be computed. Moreover, with implicit methods, the material tangent stiffness (11) needs to be computed at the end of the iterations in Algorithm 1 so that the total computational labour seems to be of the same order.

\section{Treatment of corner plasticity case}

In the discussion on the algorithms presented above, the intricacy related to the corner plasticity was not addressed. The geometry of the corner point plasticity is illustrated in Figure 3. Therein, $E_{\sigma}$ is the elastic domain and the gradients of the plastic potentials, $g_{1}$, $g_{2}$, multiplied by the elasticity matrix $\mathbf{E}$, define the regions $\Gamma_{1}, \Gamma_{12}, \Gamma_{2}$, which are characterized as follows: In region $\Gamma_{1}$, it holds for the plastic multipliers that $\lambda_{1}>0, \lambda_{2}<0$, in $\Gamma_{12}$ it holds that $\lambda_{1}>0, \lambda_{2}>0$, and in $\Gamma_{2}$ it holds that $\lambda_{1}<0, \lambda_{2}>0$ [2]. Thereby, a trial stress $\sigma_{\text {trial2 }}$ located at $\Gamma_{2}$ arrives, during the stress return mapping, at the region where $f_{1}<0$ which results in $\lambda_{1}<0$, as illustrated in Figure 3 . Thus, the genuine corner point situation and the consequent successful (i.e. with $\lambda_{1}>0, \lambda_{2}>0$ ) return mapping to the corner point can take place only when the trial stress is located in the cone $\Gamma_{12}\left(\sigma_{\text {triall }}\right.$ in Figure 3$)$.

However, according to Simo \& Hughes [2], the set of active criteria cannot be known in advance. This is because the positivity of failure criteria in the trial step does not guarantee that a genuine corner plasticity case has been realized [2], as was just illustrated. The trial-and-error type of remedy proposed in [2] is adopted here with Algorithm 1. In this method the working set of active criteria is updated during the iterative process, i.e. if either of the updated increments is negative, $\Delta \lambda_{\mathrm{vd}, n+1}<0$ or $\Delta \lambda_{\mathrm{vp}, n+1}<0$, then this criterion is dropped and the stress return mapping is restarted with the remaining criterion.

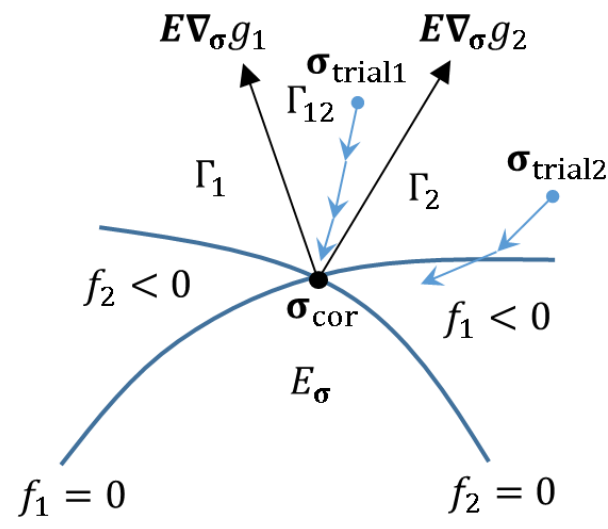

Figure 3. Geometric illustration of a corner point $\sigma_{\mathrm{cor}}$.

As to Algorithm 2 where the viscodamage and viscoplasticity integrations are performed independently, there seems to be no need for special corner point techniques. 
However, hypothetically, this same scheme could be applied with a modification: if the trial stresses, $\boldsymbol{\sigma}_{n+1}^{\mathrm{vp}, \text { trial }}=\mathbf{C}_{\mathrm{e}}:\left(\boldsymbol{\varepsilon}_{n+1}^{(i)}-\boldsymbol{\varepsilon}_{n}^{\mathrm{vp}}-\boldsymbol{\varepsilon}_{n+1}^{\mathrm{vd},(k)}\right)$ or $\boldsymbol{\sigma}_{n+1}^{\mathrm{vd}, \text { trial }}=\mathbf{D}_{n}^{-1}: \boldsymbol{\varepsilon}_{n+1}^{\mathrm{vd},(k)}$, result in a negative value of the corresponding criterion, $f_{\mathrm{vp}}^{\text {trial }}<0$ or $f_{\mathrm{vd}}^{\text {trial }}<0$, the algorithm has arrived either in $\Gamma_{1}$ or $\Gamma_{2}$ indicating that the stress integration needs could be restarted with a single criterion.

\section{Numerical examples}

\section{Model specification}

Some illustrative numerical examples are given here by applying the general theory presented above to the case of Mohr-Coulomb (MC) viscoplasticity model combined with Rankine viscodamage model. This model, assuming $k_{\mathrm{vp}}=k_{\mathrm{vd}} \equiv 1$ in (2), and (5), is specified by following equations (in the $x y$-stress space)

$$
\begin{aligned}
& f_{\mathrm{R}}\left(\boldsymbol{\sigma}, \lambda_{\mathrm{R}}, \dot{\lambda}_{\mathrm{R}}\right)=\underbrace{\frac{1}{2}\left(\sigma_{x}+\sigma_{y}\right)+\sqrt{\left(\frac{\sigma_{x}-\sigma_{y}}{2}\right)^{2}+\sigma_{x y}^{2}}}_{\hat{f}_{\mathrm{vd}}(\boldsymbol{\sigma})}-\sigma_{\mathrm{t}}\left(\lambda_{\mathrm{R}}, \dot{\lambda}_{\mathrm{R}}\right) \\
& f_{\mathrm{MC}}\left(\boldsymbol{\sigma}, \lambda_{\mathrm{MC}}, \dot{\lambda}_{\mathrm{MC}}\right)=\underbrace{\frac{k_{\varphi}-1}{2}\left(\sigma_{x}+\sigma_{y}\right)+\left(k_{\varphi}+1\right) \sqrt{\left(\frac{\sigma_{x}-\sigma_{y}}{2}\right)^{2}+\sigma_{x y}^{2}}}_{\hat{f}_{\mathrm{vp}}(\boldsymbol{\sigma})}-\sigma_{\mathrm{c}}\left(\lambda_{\mathrm{MC}}, \dot{\lambda}_{\mathrm{MC}}\right) \\
& \sigma_{\mathrm{t}}\left(\lambda_{\mathrm{R}}, \dot{\lambda}_{\mathrm{R}}\right)=\sigma_{\mathrm{t} 0}+h_{\mathrm{R}} \lambda_{\mathrm{R}}+s_{\mathrm{R}} \dot{\lambda}_{\mathrm{R}}, \quad h_{\mathrm{R}}=-g \sigma_{\mathrm{t} 0} \exp \left(-g \kappa_{\mathrm{R}}\right) \\
& \sigma_{\mathrm{c}}\left(\lambda_{\mathrm{MC}}, \dot{\lambda}_{\mathrm{MC}}\right)=\sigma_{\mathrm{c} 0}+h_{\mathrm{MC}} \lambda_{\mathrm{MC}}+s_{\mathrm{MC}} \dot{\lambda}_{\mathrm{MC}} \\
& k_{\varphi}=(1+\sin \varphi) /(1-\sin \varphi)
\end{aligned}
$$

where $\sigma_{\mathrm{t} 0}$ and $\sigma_{\mathrm{c} 0}$ are the tensile and compressive strengths, $\varphi$ is the internal friction angle, $h_{\mathrm{R}}$ and $h_{\mathrm{MC}}$ are the softening moduli in tension and compression, respectively, and $s_{\mathrm{R}}$ and $s_{\mathrm{MC}}$ are the constant viscosity moduli. The softening moduli are defined with the specific mode I and II fracture energies as $g=\sigma_{\mathrm{t} 0} l_{e} / G_{\mathrm{Ic}}$ and $h_{\mathrm{MC}}=-\sigma_{\mathrm{c} 0}{ }^{2} l_{e} / 2 G_{\text {IIc }}$ with $l_{e}$ being a characteristic length of a finite element. Moreover, non-associated flow rule with a potential, $g_{\mathrm{MC}}$, similar in form to $f_{\mathrm{MC}}$ but using the dilatation angle $\psi$ in the definition of $k_{\varphi}$ instead of the internal friction angle is employed.

\section{Uniaxial cyclic response}

First, the response of this model is demonstrated at the material point level using a computational model consisting of two CST (constant strain triangle) element model in Figure $4 \mathrm{a}$ by imposing a load reversal program in vertical $y$-direction at nodes 3 and 4 . The loading program given as displacement BC starts with a tension cycle followed by a compression cycle, and finally terminates in tension.

The material properties used for demonstrative purposes, and the model parameters used in the simulations are: Young's modulus $E=60 \mathrm{GPa}$, Poisson's ratio $v=0.2$, material density $\rho=2600 \mathrm{~kg} / \mathrm{m}^{3}$, tensile strength $\sigma_{\mathrm{t} 0}=10 \mathrm{MPa}$, compressive strength 
$\sigma_{\mathrm{c} 0}=100 \mathrm{MPa}$, mode I fracture energy $G_{\text {Ic }}=50 \mathrm{~N} / \mathrm{m}$, mode II fracture energy $G_{\text {IIc }}=$ $25 G_{\mathrm{Ic}}$, internal friction angle $\varphi=30^{\circ}$, dilatation angle $\psi=5^{\circ}$, and viscosity moduli $s_{\mathrm{R}}=$ $0.005 \mathrm{MPa} \cdot \mathrm{s}, s_{\mathrm{MC}}=0.005 \mathrm{MPa} \cdot \mathrm{s}$.

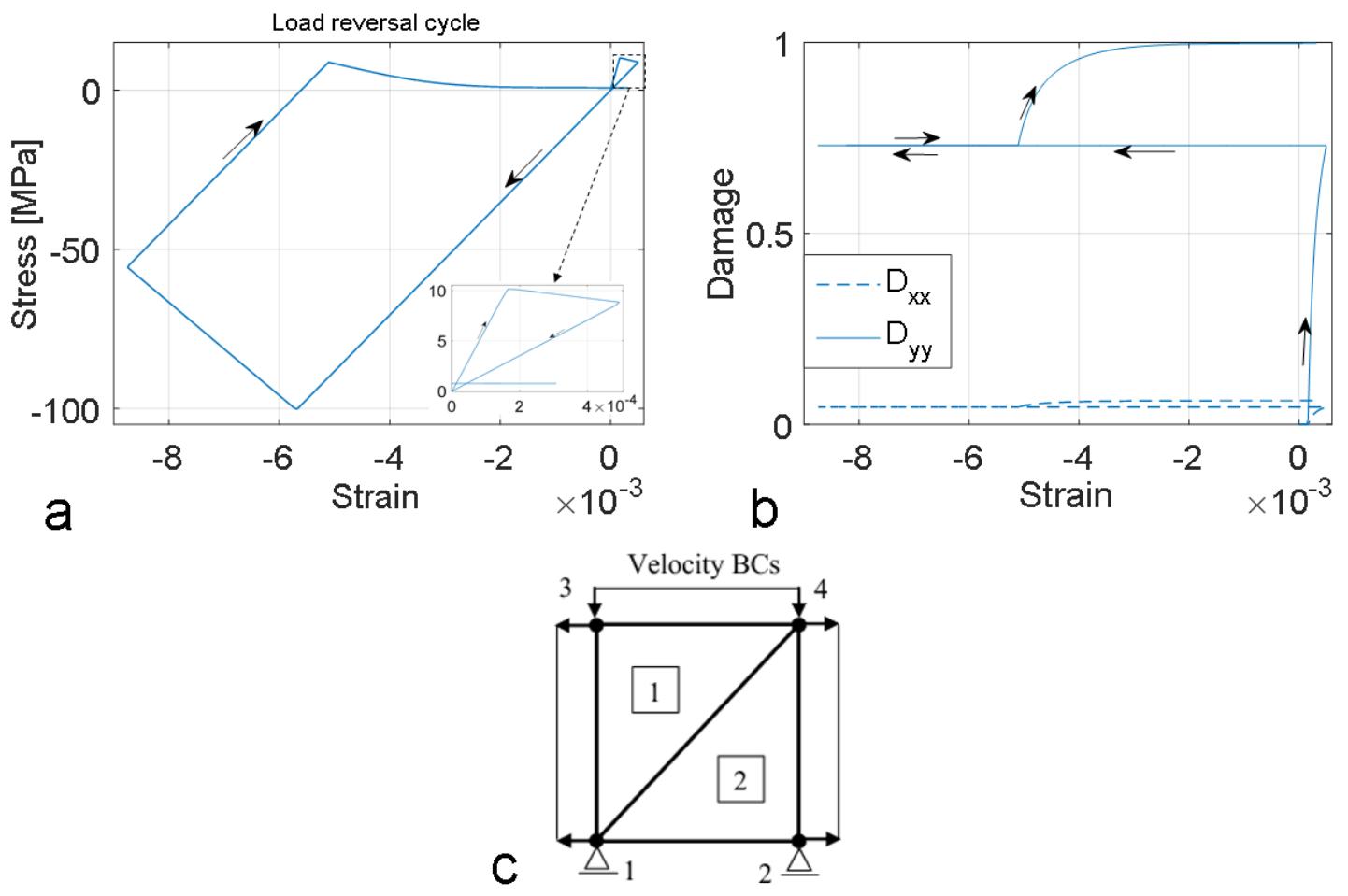

Figure 4. Model prediction with two-element mesh in cyclic loading: stress-strain response (a), damage component evolution (b), and the computational model (c).

As the objective of the present paper is to compare the stress integration methods and not to model any real rock, these properties are chosen so as to represent a rock-like material in general, not any particular real rock.

Figure $3 \mathrm{~b}$ shows the corresponding damage development where the damage components are calculated by $D_{i i}=1-E_{\mathrm{d}, i i} / E_{0, i i}$ where $E_{\mathrm{d}, i i}$ and $E_{0, i i}$ are the diagonal entries of damage degraded and intact stiffness tensor, respectively. It can be noticed that stiffness degrades during the first tension cycle but no irreversible strain is generated. During the compression cycle, viscoplastic strains develop but no stiffness degradation. The reader should note that unilateral conditions of damage, i.e. the stiffness recovery upon crack closure in compression, are not accounted for in the present model as mentioned above. Finally, it should be reminded that both methods give the same results here since the corner point plasticity does not occur with the uniaxial load reversal program used here.

\section{Bi-axial tension/compression test}

Next, the biaxial compression/tension test is simulated with the setup described in Figure 4c. Three cases of the axial and lateral velocities are considered here: the axial 
velocity is set to $v_{\mathrm{y}}=-0.05 \mathrm{~m} / \mathrm{s}$ (compression) for each case while the lateral velocities are $v_{\mathrm{x}}=0.0125 \mathrm{~m} / \mathrm{s}, v_{\mathrm{x}}=0.025 \mathrm{~m} / \mathrm{s}$, and $v_{\mathrm{x}}=0.05 \mathrm{~m} / \mathrm{s}$ (tension) for cases $1,2,3$, respectively. The results for stress-strain responses are shown in Figure 5.
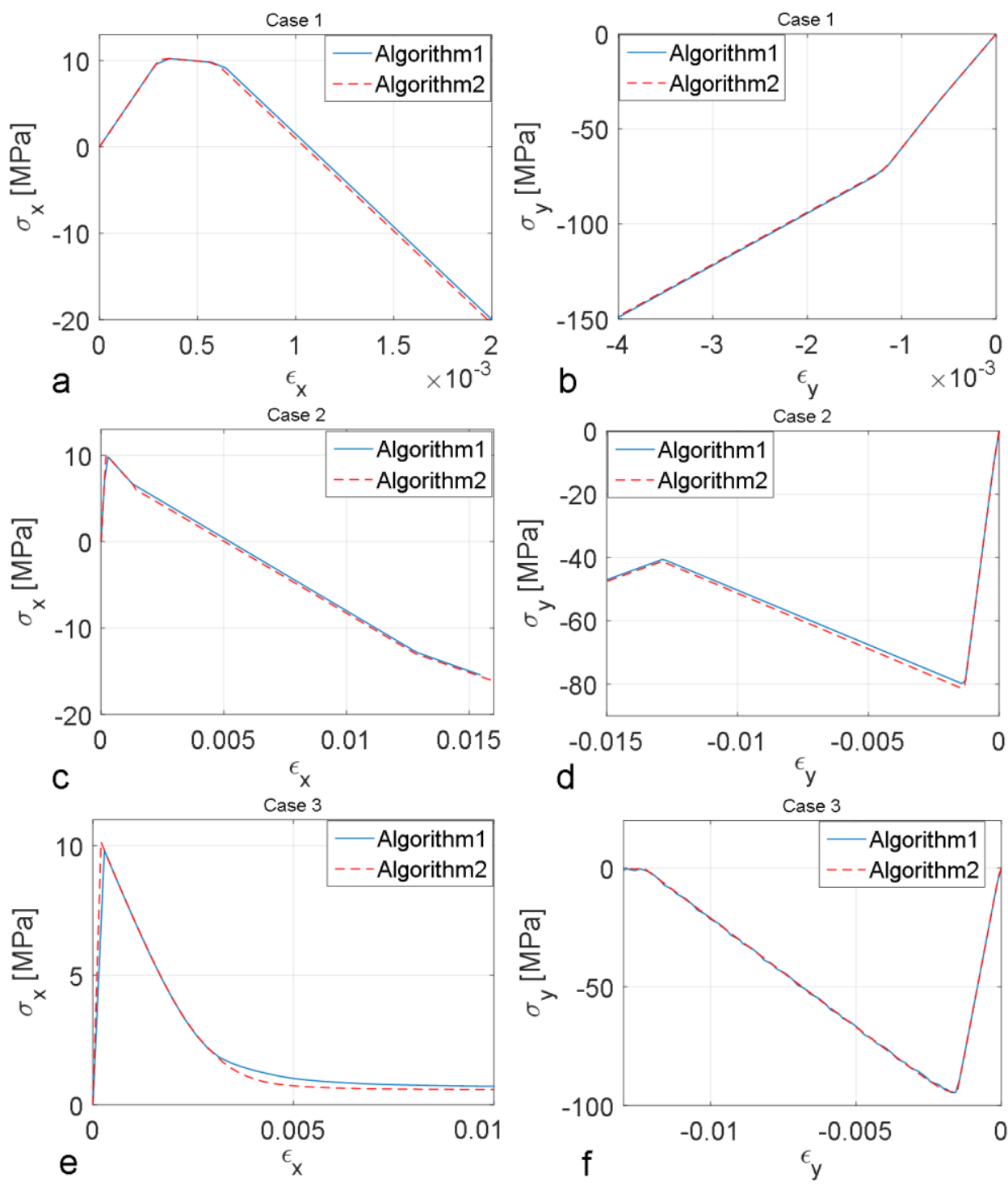

Figure 5. Model with the two-element model in bi-axial tension/compression: stress-strain curves with $v_{\mathrm{x}}=0.0125 \mathrm{~m} / \mathrm{s}$ (a), (b), $v_{\mathrm{x}}=0.025 \mathrm{~m} / \mathrm{s},(\mathrm{c}),(\mathrm{d})$, and $v_{\mathrm{x}}=0.05 \mathrm{~m} / \mathrm{s}$ (e), (f).

In Case 1, i.e. the lowest lateral velocity, the Rankine type of damage process is activated first in lateral direction. Later on, as the compressive strength in mixed tension compression is reached, the return type changes to a return on the MC surface. The resulting stress-strain responses for both model are shown in Figure 5a and b. In Case 2, the lateral velocity being $0.025 \mathrm{~m} / \mathrm{s}$ in both negative and positive $x$-direction results in equibi-axial tension/compression. The realized return types are the same as in Case 1 but a notable difference in the stress-strain responses is that the axial stress show a softening response, in contrast to hardening one in Case 1, as attested in Figure 5d. 
Finally, in Case 3, the lateral extension velocity is high enough to change the return type to the corner point case. This change is reflected in the stress-strain response so that both the $x$ and $y$ components of the stress reach, as the corner point moves towards the origin of the stress space due to softening, the residual values during the loading process (Figure $5 \mathrm{e}$ and $\mathrm{f}$ ). It should be mentioned here that Algorithm 2 required three iterations to return the trial stress into the corner point with the convergence tolerance of $10 \mathrm{E}-12$.

As to Algorithm 1 in Case 3, it did not reach a stable corner point return with the present parameter values. Instead, an unstable behaviour "oscillating" between a return to MC and Rankine surfaces was attested.

\section{Conclusions}

Two stress integration (return mapping) algorithms for coupled viscodamageviscoplasticity models were considered in this paper. The first was the classical direct solution of the plastic increments and the consequent trial stress correction with a formula similar to the Koiter's rule. The second, far less standard, algorithm integrates the viscodamage and viscoplasticity problems independently and then imposes the stress equality iteratively adjusting the damage strain.

The general theory was applied to coupled Rankine visco-damage-Mohr-Coulomb viscoplasticity softening model. The numerical examples showed that the latter, nonstandard, algorithm is robust and converges fast in genuine corner point return types. Therefore, it deserves to be more widely applied in computational structural failure analyses

\section{Acknowledgements}

This research was funded by the Academy of Finland (Grant no. 298345).

\section{References}

[1] J. Lemaitre. A Course on Damage Mechanics. Springer-Verlag, 1990.

[2] J.C. Simo, T.J.R. Hughes. Computational Inelasticity. Springer, 2000.

[3] A. Ibrahimbegovic. Nonlinear solid mechanics: theoretical formulations and finite element solution methods. Berlin: Springer; 2009.

[4] P. Grassl, M. Jirásek. Damage-plastic model for concrete failure. International Journal of Solids and Structures. 43: 7166-7196, 2006. https://doi.org/10.1016/j.ijsolstr.2006.06.032

[5] E. Hansen, K. Willam, I. Carol. A two-surface anisotropic damage/plasticity model for plain concrete. In: de Borst R, et al., editors. Proceedings of Framcos4 conference Paris (fracture mechanics of concrete structures). Rotterdam: Balkema; 2001.

[6] A. Ibrahimbegovic, D. Markovic, F. Gatuingt. Constitutive Model of Coupled Damage-Plasticity and Its Finite Element Implementation. Revue européenne des éléments finis. 12, 381-405, 2003. 
[7] T. Saksala, A. Ibrahimbegovic. Anisotropic viscodamage-viscoplastic consistency constitutive model with a parabolic cap for rocks with brittle and ductile behaviour. International Journal of Rock Mechanics \& Mining Sciences. 70:460 473, 2014. https://doi.org/10.1016/j.ijrmms.2014.05.019

[8] F. Armero, S. Oller. A general framework for continuum damage models. I. Infinitesimal plastic damage models in stress space. International Journal of Solids and Structures. 37:7409-7436, 2000. https://doi.org/10.1016/S00207683(00)00205-5

[9] R.K. Abu Al-Rub, G.Z. Voyiadjis. On the coupling of anisotropic damage and plasticity models for ductile materials. International Journal of Solids and Structures. 40: 2611-2643, 2003. https://doi.org/10.1016/S0020-7683(03)00109-4

[10] W.M. Wang, L.J. Sluys, R. De Borst. Viscoplasticity for instabilities due to strain softening and strain-rate softening. International Journal of Numerical Methods in Engineering. 40: 3839-64, 1997. https://doi.org/10.1002/(SICI)10970207(19971030)40:20<3839::AID-NME245>3.0.CO;2-6

Timo Saksala

Department of Civil Engineering, Tampere University of Technology P.O. Box 600, FI-33101 Tampere

timo.saksala@tut.fi 